\title{
ATLAS IBL operational experience
}

\author{
Yosuke Takubo on behalf of the ATLAS collaboration* \\ High Energy Accelerator Research Organization (KEK) \\ 1-1 Oho Tsukuba Ibaraki, 305-0801, Japan \\ E-mail: yosuke.takubo@kek.jp
}

\begin{abstract}
The Insertable B-Layer (IBL) is the inner most pixel layer in the ATLAS experiment, which was installed at a $3.3 \mathrm{~cm}$ radius from the beam axis in 2014 to improve the tracking performance. To cope with the high radiation and hit occupancy due to proximity to the interaction point, a new read-out chip and two different silicon sensor technologies (planar and 3D) have been developed for the IBL. After the long shut-down period over 2013 and 2014, the ATLAS experiment started data-taking in May 2015 for Run-2 of the Large Hadron Collider (LHC). The IBL has been operated successfully since the beginning of Run- 2 and shows excellent performance with a low dead module fraction, high data-taking efficiency and improved tracking capability. The experience and challenges in operation of the IBL are described and results on the performance are presented.
\end{abstract}

The 25th International workshop on vertex detectors

September 26-30, 2016

La Biodola, Isola d' Elba, ITALY

${ }^{*}$ Speaker. 


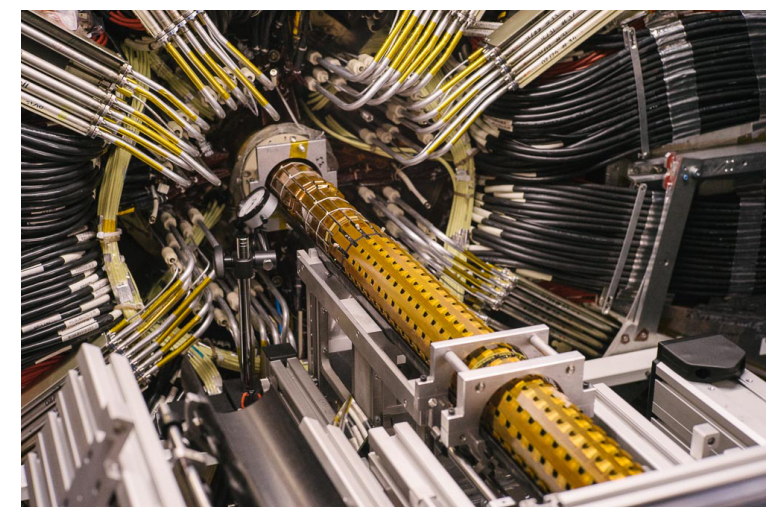

Figure 1: Picture of IBL insertion into the ATLAS detector.

\section{Introduction}

The Pixel Detector used in Run-1 is designed to work at the instantaneous luminosity of $1 \times$ $10^{34} \mathrm{~cm}^{-2} \mathrm{~s}^{-1}$. The instantaneous luminosity of the Large Hadron Collider (LHC) is expected to 4 increase up to $3 \times 10^{34} \mathrm{~cm}^{-2} \mathrm{~s}^{-1}$ after long shutdown 2 (LS2) which is planned in 2019. As the 5 luminosity increases the hit data rate becomes a serious issue for the front-end electronics. To cope 6 with the increase during Run-2, a new pixel sensor layer, IBL [1], was installed as the inner most layer in 2014. This was the first upgrade project in the ATLAS detector [2].

The installation of the IBL was strongly motivated by physics analysis as well. For example, $b$-jet tagging is used in about $65 \%$ of the analysis results published by the ATLAS collaboration at the ICHEP conference in 2016 [3]. Providing additional hit information at the closest position to the beam collision point, the IBL significantly improves the performance of $b$-jet tagging.

After the long shut-down period over 2013 and 2014 (LS1), the ATLAS experiment started data-taking in May 2015 for Run- 2 of the LHC. The IBL has been successfully operated and shows excellent performance. In the following sections, the experience and challenges in the operation of the IBL are described as well as its performance.

\section{Insertable B-Layer (IBL)}

In the IBL, two different sensor technologies are adopted: $\mathrm{n}^{+}$-in-n planar sensors [4] and 3D sensors [5]. The pixel size of the sensors is $50 \times 250 \mu \mathrm{m}^{2}$ which is $60 \%$ of the pixel size used for the Pixel Detector $\left(50 \times 400 \mu \mathrm{m}^{2}\right)$. The IBL has about 12 million pixels in total. The thickness of the planar and 3D sensors is $200 \mu \mathrm{m}$ and $230 \mu \mathrm{m}$, respectively. In the planar sensor, the slimedge of $200 \mu \mathrm{m}$ with long pixels under the guard-ring is adopted to minimize inactive region. The sensors were designed to work at a fluence of up to $5 \times 10^{15} 1 \mathrm{MeV} \mathrm{n}_{\mathrm{eq}}$, which is the expected fluence by the end of LHC Phase-1 operation around 2022.

A new front-end chip (FE-I4B) was developed for the IBL. The FE-I4B is produced with IBM $130 \mathrm{~nm}$ technology, and $80 \times 336$ pixels are embedded per chip. It is designed to be radiation hard up to a $250 \mathrm{Mrad}$ ionizing dose. The signals from the sensor are amplified and digitized in analog and digital circuits respectively, and finally the charge information is output with 4-bit value of the time over threshold (TOT). 


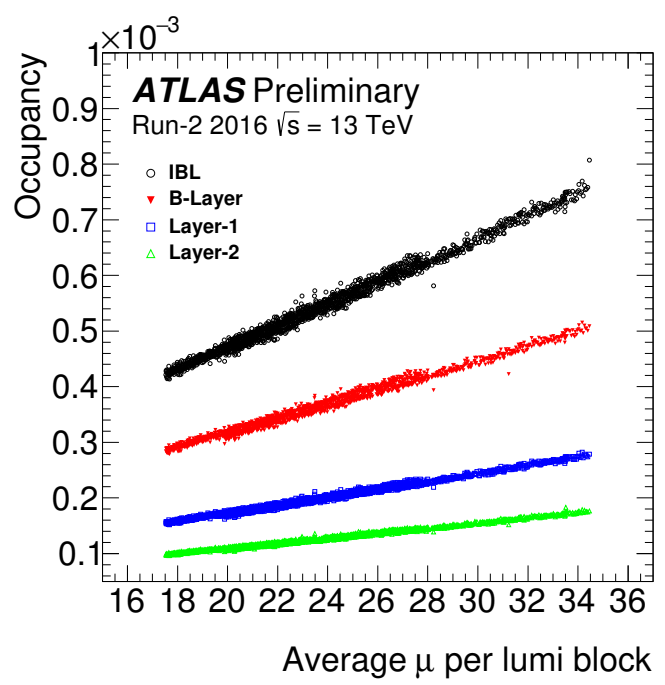

Figure 2: Hit occupancy at each pixel layer as a function of the number of pile-up events $(\mu)$. IBL, B-Layer, Layer-1 and Layer-2 are placed at the radius of 33, 50.5, 88.5 and $122.5 \mathrm{~mm}$, respectively, centered around the beam axis [6].

An IBL module consists of a sensor bump-bonded to an FE-I4B chip with flex cables which provide electric lines. The modules are mounted on the staves where planar sensors are used for $75 \%$ of the middle region in $\eta$ and 3D sensors used in the remaining $25 \%$ (high $\eta$ ). The full IBL consists of 14 staves integrated into a support tube. The IBL surrounds a new beam pipe, and is contained in a package of $7 \mathrm{~m}$ long in the limited radial envelope of $10 \mathrm{~mm}$. The IBL was transported to the ATLAS experimental hall $90 \mathrm{~m}$ underground on May 5, 2014 and installed into the ATLAS detector on May 7 as shown in Fig. 1. To install the IBL in the limited space inside B-Layer, an insertion clearance of less than $0.1 \mathrm{~mm}$ was available.

\section{Performance of IBL in ATLAS Run-2 operation}

The ATLAS experiment started data-taking in May 2015 for Run- 2 of the LHC. The IBL has been operating successfully since the beginning of Run-2 and shows excellent performance with low dead module fraction, high data-taking efficiency and improved tracking capability as summarized in this section.

\subsection{Detector stability in data-taking}

One of the big achievements in the IBL operation is that $100 \%$ of the sensors and FE-I4B chips are kept operational in the tracking coverage region of $|\eta|<2.5$ during Run-2. It allows the IBL to show full tracking performance.

Figure 2] [6] shows the hit occupancy at each pixel layer as a function of the number of pile-up events $(\mu)$. The hit occupancy at the IBL increases linearly with $\mu$, and has been successful even at high $\mu$. Actually, the rate of desynchronization in the readout is of the order of $0.1 \%$ at the IBL and this value is about one order of magnitude less than those of the other Pixel layers. 

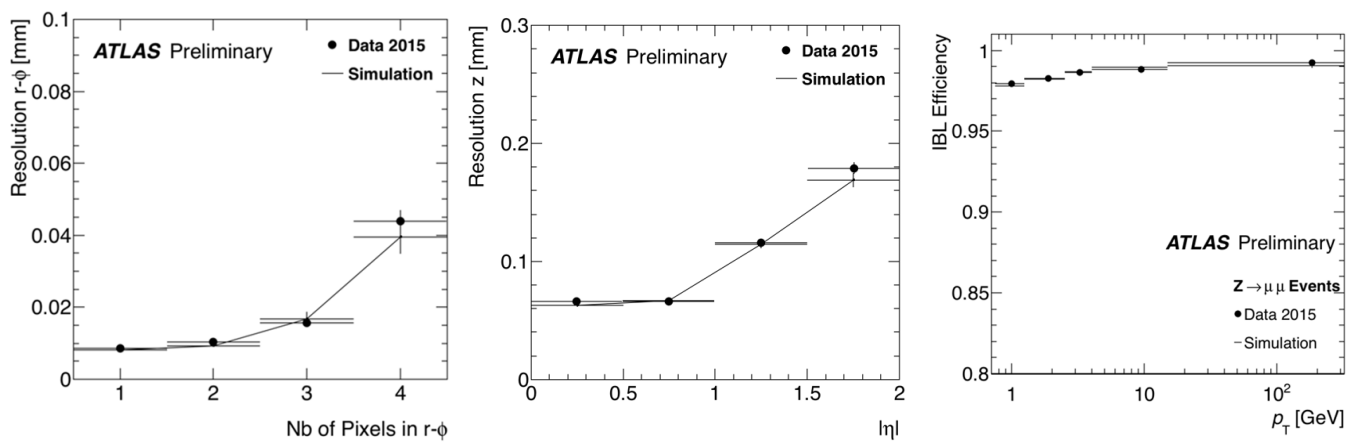

Figure 3: The hit position resolution on the IBL in the $r-\phi$ (left) and $z$ (middle) projections as a function of cluster width along $\phi$ and the track $|\eta|$, respectively, and efficiency of associating an IBL hit to a reconstructed particle track as a function of the particle $p_{\mathrm{T}}$ (right) 7 . The selected $Z \rightarrow \mu \mu$ events in collision data (circles with error bars) and simulation (lines) are used for these plots.
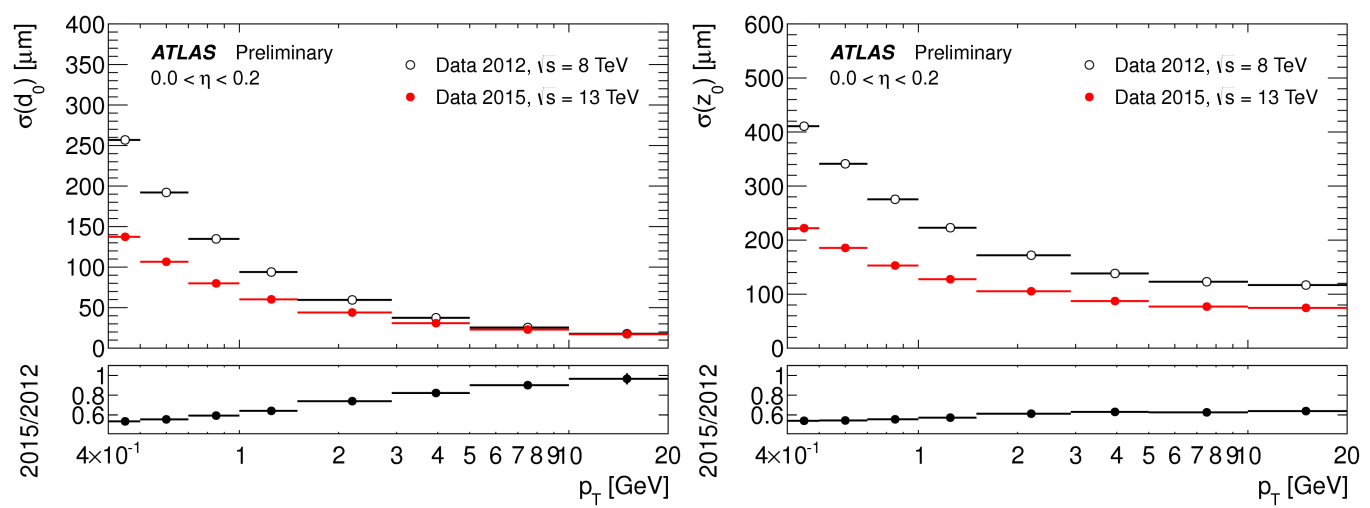

Figure 4: $d 0$ (left) and $z 0$ resolution (right) as a function of $p_{\mathrm{T}}$ in ATLAS Run-1 (without IBL) and Run-2 (with IBL) [8].

\subsection{Position resolution and hit efficiency}

Figure 3 [7] shows the hit position resolution on the IBL in the $r-\phi$ (left) and $z$ (middle) projections as a function of cluster width along $\phi$ and the track $|\eta|$, respectively. In Fig. 3 (left), the clusters that are broader than two pixels along the $r-\phi$ projection typically include secondary ionization and thus exhibit a degraded hit spatial resolution along that coordinate, mostly due to the effect of $\delta$-rays, as predicted by simulation. The evolution of the measured resolution along the longitudinal coordinate in Fig. 3 (middle) is interpreted as a convolution of the change in resolution with the increasing number of pixels used for charge interpolation and the increase of the multiple scattering effects.

Figure 3 (right) [7] shows efficiency of associating an IBL hit to a reconstructed particle track as a function of the particle $p_{\mathrm{T}}$. The efficiency is computed as the fraction of selected tracks with $p_{\mathrm{T}}>0.7 \mathrm{GeV}$ and $|\eta|<2.5$ that have an associated IBL cluster. The efficiency is better than $98 \%$ and is measured as expected by the MC simulation.

The IBL provides the hit information at the closest position to the beam collision point, and it improves the impact parameter resolution. Figure 4 [8] shows $d 0$ and $z 0$ resolution in Run-1 


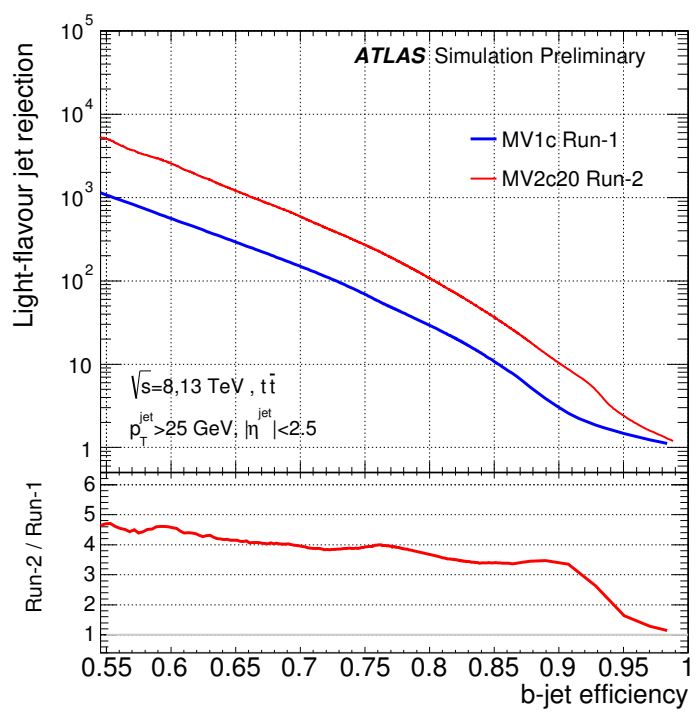

Figure 5: The light jet rejection versus $b$-jet tagging efficiency for the MV1c $b$-tagging algorithm using the Run-1 detector and reconstruction software (blue) compared to the MV2c20 $b$-tagging algorithm using the Run-2 setup (red) [9].

and Run-2. The impact parameter resolutions are better in Run-2 with the IBL. At low transverse momentum $\left(p_{\mathrm{T}}\right)$ region, and it is improved by about $40 \%$ at $p_{\mathrm{T}}$ below $1 \mathrm{GeV}$ for both $d 0$ and $z 0$.

\section{$3.3 b$-jet tagging performance}

The better impact parameter resolution with the IBL improves performance of $b$-jet tagging. Figure 5 shows simulation results for two software algorithms: MV1c and MV2c20. The light jet rejection as a function of $b$-jet tagging efficiency for MV1c $b$-tagging algorithm using the Run-1 detector and reconstruction software (blue) compared to the MV2c20 $b$-tagging algorithm using the Run-2 setup [9]. The light jet rejection shows better performance with the IBL together with improvements in the algorithm of $b$-jet tagging.

\section{4 $d E / d x$ measurement}

$d E / d x$ information is important especially for physics analysis to find new heavy particles predicted in physics beyond the Standard Model [10]. The IBL gives another measurement of charge deposition in the pixel sensors which improves the $d E / d x$ measurement. Figure 6 [11] shows a distribution of $d E / d x$ obtained with (black dots) and without the IBL (red triangles) (left) and bi-dimensional distribution of $\mathrm{dE} / \mathrm{dx}$ and momentum (right). It can be seen that the distribution of $d E / d x$ in Fig. 6 (left) becomes narrower with the IBL. In addition, the particle types are clearly separated in Fig. 6(right), and this information is used for particle identification in physics analyses.

\section{Challenges in IBL operation}

The IBL has faced several challenges like wire-bond oscillation, distortion of the staves with changing temperature and low-voltage (LV) current increase due to total ionizing dose (TID) effect. 

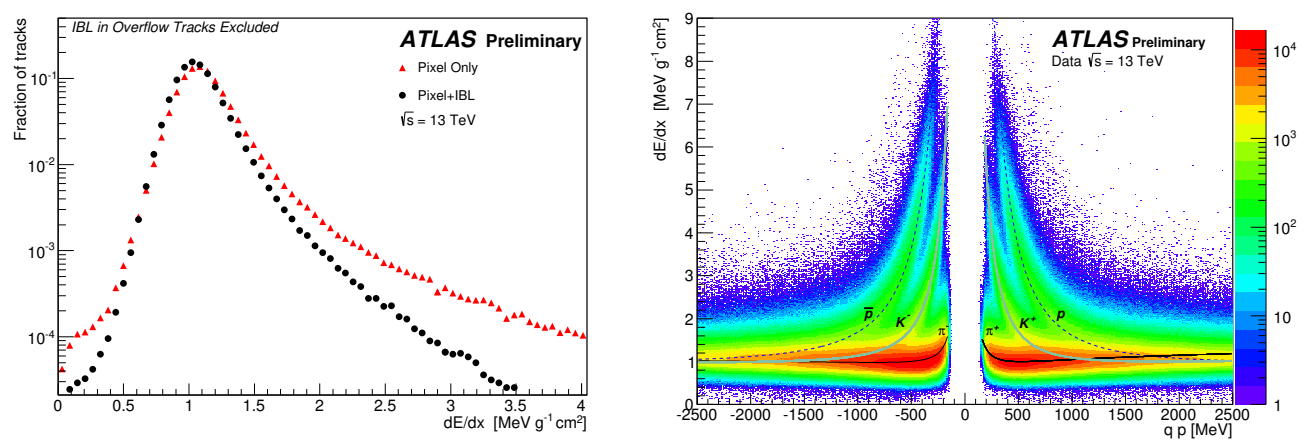

Figure 6: Distribution of $d E / d x$ obtained with (black dots) and without the IBL (red triangles) (left) and bidimensional distribution of $d E / d x$ and momentum (right) [11]. In the right plot, the distributions of the most probable value for the fitted probability density functions of pions, kaons and protons are superimposed.

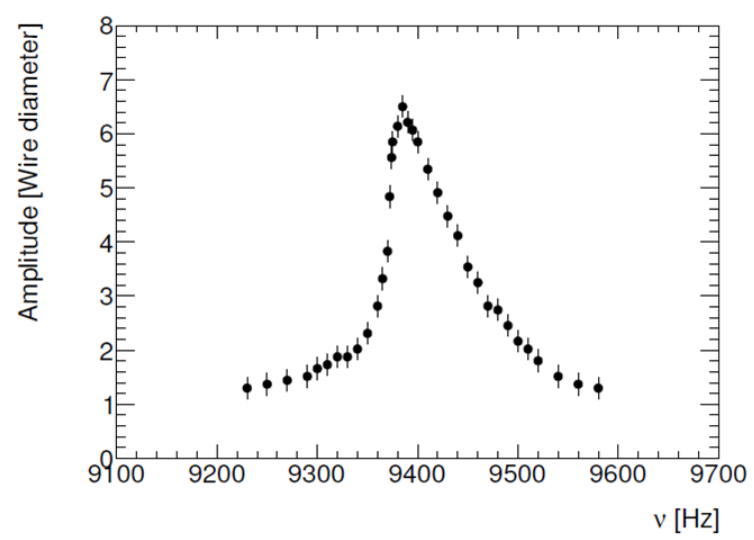

Figure 7: Oscillation amplitude expressed in wire diameter $(25 \mu \mathrm{m})$ as a function of the frequency for a wire of $2.8 \mathrm{~mm}$ length obtained under IBL-like conditions [12].

Coping with these challenges, the detector has been successfully operated in stable condition during Run-2 in 2015 and 2016. In this section, the challenges faced in the operation and the treatment for them are described.

\subsection{Wire bond oscillation}

Wire bonding is used to connect the FE-I4B chip to the flex cables on the IBL module. The digital circuit of the FE-i4B chip consumes current which is susceptible to consecutive triggers or calibration scans [12]. Accordingly, the wire bonds may oscillate at relatively high frequency in the magnetic field of $2 \mathrm{~T}$ where the IBL is located. Figure 7 shows the oscillation amplitude as a function of the frequency obtained experimentally for a $2.8 \mathrm{~mm}$ wire under IBL-like conditions. Depending on the oscillation amplitude and number of cycles, micro-cracks can develop at the wire bond heal and, even more, the wire can cross the material elastic limit. Both aspects may lead to possible failures on the bond.

To mitigate this effect, the trigger cycle sent to the FE-I4B chip was manipulated to avoid the resonance frequency of the wire bonds. In operation of the IBL, a fixed frequency trigger 


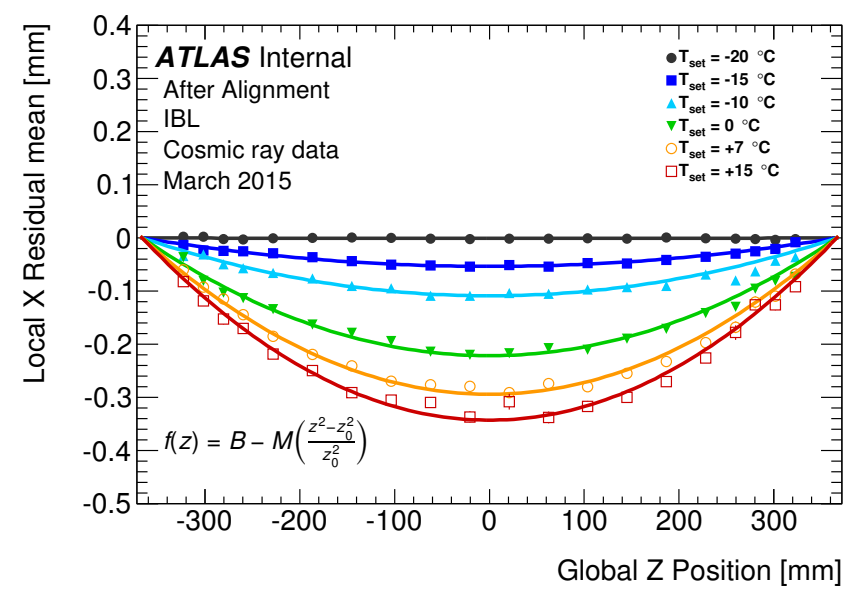

Figure 8: The track-to-hit residual mean in the local $x$ direction [13]. The residual mean is averaged over all hits of modules at the same global $z$ position. The alignment corrections derived at $-20{ }^{\circ} \mathrm{C}$ are applied to the local positions in the module frames.

veto (FFTV) was newly implemented in data-taking system to limit the number of triggers in the resonance region.

\subsection{Mechanical distortion of IBL staves}

During the commissioning of the IBL, distortion of the staves was found [13]. The magnitude of the distortion depends on the operating temperature as shown in Fig. 8 and it is caused by a mismatch between the coefficients of thermal expansion (CTE) of a bare stave made with the carbon foam and the flex cable attached on the bare stave. The maximum displacement due to the distortion is more than $300 \mu \mathrm{m}$ at the planned operating temperature of $-20^{\circ} \mathrm{C}$ with respect to the nominal position at the room temperature.

In the operation of the IBL, the impact of this effect is mitigated by temperature control at the level of $0.2 \mathrm{~K}$ and the regular alignment correction in the offline reconstruction.

\subsection{Effect of total ionizing dose on FE-I4B}

The LV current consumption in FE-I4B chips increased in 2015. This was caused by the effect of TID on NMOS transistors in the chip. Significant leakage current was induced by positive charges trapped in the bulk of the shallow trench isolation (STI) in NMOS transistor [14].

The increase of the leakage current causes several issues in the detector operation: In the worst case, and if only one power wire bond is left, the IBL module approaches the maximum LV current of $0.55 \mathrm{~A}$. In 2015 the current started to approach this limit before mitigating actions were taken. In addition, it was observed that this phenomenon causes detuning of ToT and threshold values [15]. The power consumption in a FE-I4B increases with more LV current, and it changes temperature on the module. As described in Sec. 4.2 the different temperature on the modules causes distortion of the staves accordingly.

To study this effect as a function of TID the measurement of LV current in a FE-I4B chip was performed after irradiating it with an x-ray source and proton beam [16, 17]. The results showed 


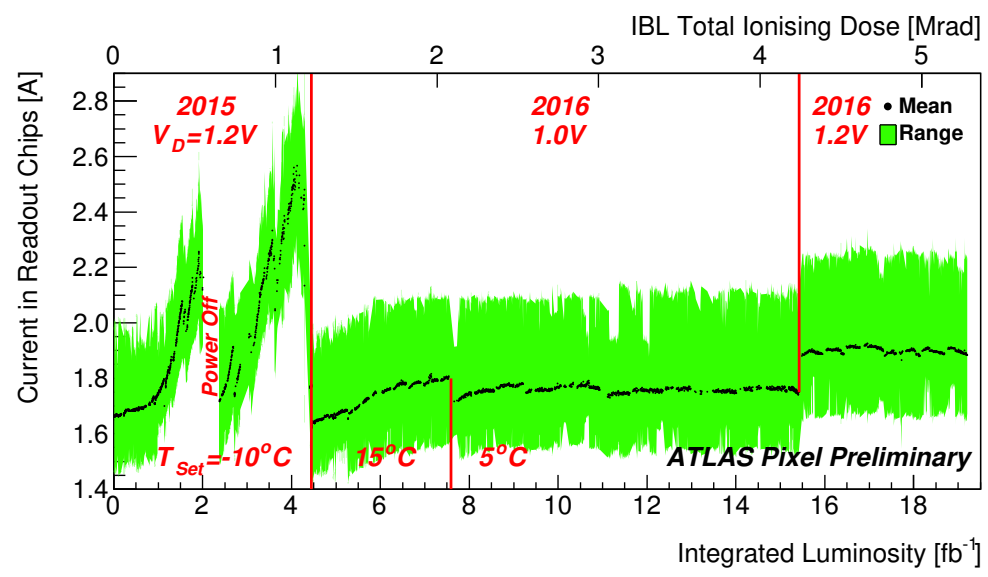

Figure 9: Mean LV current of four FE-I4B chips in one LV channel group against integrated luminosity and TID during Run-2 [18].

that LV current increases until about $1 \mathrm{Mrad}$ where it begins to decrease again [16]. This can be explained by the interface traps filling with electrons in front of STI after the positive charge has been trapped there. This layer of negative charge compensates for the effect of trapped holes in the STI. In addition, it was found that the LV current can be decreased by operating the chip at higher temperature due to the annealing effect [16].

Taking into account the results of these measurements, the operating temperature of the IBL was increased to $15^{\circ} \mathrm{C}$ at the beginning of 2016 from $-10^{\circ} \mathrm{C}$ in 2015 and then eventually decreased again to $5{ }^{\circ} \mathrm{C}$. In addition, the digital supply-voltage was decreased to $1.0 \mathrm{~V}$ from $1.2 \mathrm{~V}$ until the TID was more than $4 \mathrm{Mrad}$. Figure 9[18] shows the mean LV current of four FE-I4B chips in one LV channel group against integrated luminosity and TID. Although there were significant increases in LV current during 2015, it became stabilized and contained in 2016.

\section{Summary and conclusions}

The IBL is the inner most pixel layer in the ATLAS experiment, which was installed at a 3.3 $\mathrm{cm}$ radius from the beam axis in 2014 to improve the tracking performance. After LS1, the ATLAS experiment started data-taking in May 2015 for Run-2 of the LHC. The IBL has been operated successfully since the beginning of Run-2 and shows excellent performance with the low dead module fraction, high data-taking efficiency and improved tracking capability.

The additional hit information provided by the IBL at the closest position to the beam collision point provides invaluable information. It significantly improves the tracking performance, $b$-jet tagging and $d E / d x$ measurements for example.

Operating the IBL has faced several challenges like wire-bond oscillation, distortion of the staves with changing temperature and LV current increase due to TID effect. Coping with these challenges, the detector has been successfully operated in stable conditions during Run- 2 in 2015 and 2016. 


\section{References}

[1] The ATLAS Collaboration, ATLAS Insertable B-Layer Technical Design Report, CERN-HLCC-2010-013, ATLAS TDR 19, 15 September 2010.

[2] ATLAS Collaboration, The ATLAS Experiment at the CERN Large Hadron Collider, JINST 3 S08003, 2008.

[3] 38th INTERNATIONAL CONFERENCE ON HIGH ENERGY PHYSICS (ICHEP 2016), https://www.ichep2016.org.

[4] C. Goessling et al., Planar $n^{+}$-in- $n$ silicon pixel sensors for the ATLAS IBL upgrade, Nucl. Instrum. Meth. A 650 (2011), 198-201.

[5] Cinzia Da Via et al., 3D silicon sensors: Design, large area production and quality assurance for the ATLAS IBL pixel detector upgrade, Nucl. Instrum. Meth. A 694 (2012), 321-330.

[6] ATLAS Collaboration, Public Pixel Tracker Plots for Collision Data, PIX-2016-007, http://atlas.web.cern.ch/Atlas/GROUPS/PHYSICS/PLOTS/PIX-2016-007.

[7] The ATLAS Collaboration, Efficiency and Hit Spatial Resolution of ATLAS IBL Sensors in LHC Run 2 Collision Events, ATL-INDET-PUB-2016-001, http://cds.cern.ch/record/2203893.

[8] The ATLAS Collaboration, Approved Plots of the Tracking Combined Performance Group, INTR-2015-007, http://atlas.web.cern.ch/Atlas/GROUPS/PHYSICS/PLOTS/IDTR-2015-007.

[9] The ATLAS Collaboration, Expected performance of the ATLAS b-tagging algorithms in Run-2, ATL-PHYS-PUB-2015-022, http://cds.cern.ch/record/2037697.

[10] The ATLAS Collaboration, Search for metastable heavy charged particles with large ionization energy loss in pp collisions at $\sqrt{s}=13$ TeV using the ATLAS experiment, Phys. Rev. D 93, 112015 (2016).

[11] The ATLAS Collaboration, Public Pixel Tracker Plots for Collision Data, PIX-2015-002, http://atlas.web.cern.ch/Atlas/GROUPS/PHYSICS/PLOTS/PIX-2015-002.

[12] D. A. Feito, A. Honma, B. Mandelli, Studies of IBL wire bonds operation in a ATLAS-like magnetic field, PH-EP-Tech-Note-2015-002, http://cds.cern.ch/record/2010249.

[13] The ATLAS Collaboration, Study of the mechanical stability of the ATLAS Insertable B-Layer, ATL-INDET-PUB-2015-001, http://cds.cern.ch/record/2022587.

[14] L. Gonella et al., Total Ionizing Dose effects in 130-nm commercial CMOS technologies for HEP experiments, Nucl. Instrum. Meth. A 582 (2007), 750-654.

[15] The ATLAS Collaboration, Cosmics and Calibration Approved Pixel Plots, PIX-2016-005, http://atlas.web.cern.ch/Atlas/GROUPS/PHYSICS/PLOTS/PIX-2016-005.

[16] The ATLAS Collaboration, Cosmics and Calibration Approved Pixel Plots, PIX-2015-008, http://atlas.web.cern.ch/Atlas/GROUPS/PHYSICS/PLOTS/PIX-2015-008.

[17] The ATLAS Collaboration, Cosmics and Calibration Approved Pixel Plots, PIX-2016-010, http://atlas.web.cern.ch/Atlas/GROUPS/PHYSICS/PLOTS/PIX-2016-010.

[18] The ATLAS Collaboration, Cosmics and Calibration Approved Pixel Plots, PIX-2016-006, http://atlas.web.cern.ch/Atlas/GROUPS/PHYSICS/PLOTS/PIX-2016-006. 